Commun. Fac. Sci. Univ. Ank. Ser. A1 Math. Stat.

Volume 69, Number 1, Pages 771-781(2020)

DOI: $10.31801 /$ cfsuasmas. 680388

ISSN 1303-5991 E-ISSN 2618-6470

http://communications.science.ankara.edu.tr/index.php?series=A1

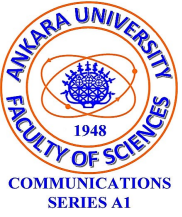

\title{
GRAND LORENTZ SEQUENCE SPACE AND ITS MULTIPLICATION OPERATOR
}

\author{
OĞUZ OĞUR
}

\begin{abstract}
In this paper, we introduce the grand Lorentz sequence spaces $\ell_{p, q)}^{\theta}$ and study on some topological properties. Also, we characterize some properties of the multiplication operator, such as compactness, Fredholmness etc., defined on $\ell_{p, q)}^{\theta}$.
\end{abstract}

\section{INTRODUCTION}

Let $(X, S, \mu)$ be a $\sigma$-finite measure space and let $g$ be a complex-valued measurable function defined on $X$. The non-increasing rearrangement $g^{*}$ of $g$ is defined by

$$
g^{*}(s)=\inf \left\{t>0: F_{\mu}(t) \leq s\right\}, \quad s \geq 0,
$$

where $F_{\mu}(t)=\mu\{x \in X:|g(x)|>t\}, t \geq 0$, is the distribution function of $g$. If $\mu$ is counting measure on $S=2^{\mathbb{N}}$, then we can write the distribution function and the non-increasing rearrangement of a complex-valued sequence $\left(x_{n}\right)$, respectively, as follows;

$$
F_{\mu}(t)=\mu\left\{n \in \mathbb{N}:\left|x_{n}\right|>t\right\}, t \geq 0
$$

and

$$
x_{\phi(n)}=\inf \left\{t>0: F_{\mu}(t) \leq n-1\right\}
$$

if $n-1 \leq t<n$ with $F_{\mu}(t)<\infty$. By the definition of non-increasing rearrangement, we can interpret that $\left(x_{\phi(n)}\right)$ can be obtained by permuting $\left(\left|x_{n}\right|\right)_{n \in R}$, where $R=$ $\left\{n \in \mathbb{N}: x_{n} \neq 0\right\}$, in the decreasing order. Here, $x_{\phi(n)}=0$ for $n>\mu(R)$ if $\mu(R)<$ $\infty$ [.

Received by the editors: January 27, 2020, Accepted: February 18, 2020.

2010 Mathematics Subject Classification. 47B38, 46E30, 46A45.

Key words and phrases. Grand Lorentz sequence spaces, multiplication operator, compactness, Fredholm operator.

(C) 2020 Ankara University Communications Faculty of Sciences University of Ankara-Series A1 Mathematics and Statistics 
Lorentz introduced the classical Lorentz space $\Lambda_{q, w}, 0<q<\infty$, which the space of all measurable functions $f$ defined on $(0,1)$ with

$$
\|f\|_{\Lambda_{q, w}}=\left(\int_{0}^{1}\left(f^{*}(x)\right)^{q} w(x) d x\right)^{\frac{1}{q}}
$$

where $f^{*}$ is the non-increasing rearrangement of $f$ and $w$ is a weight function [12, 13. The space $\Lambda_{q, w}$ and its special case $L^{p, q}, 0<q, p \leq \infty$, have been widely studied by many authors. For more details see [3], [5], [7].

The Lorentz sequence spaces $\ell_{p, q}$ is the space of all complex-valued sequences $x=\left(x_{n}\right)$ such that

$$
\|x\|_{p, q}=\left\{\begin{array}{cc}
\left(\sum_{n=1}^{\infty} n^{\frac{q}{p}-1}\left(x_{\phi(n)}\right)^{q}\right)^{\frac{1}{q}}, & 1 \leq p \leq \infty, 1 \leq q<\infty \\
\sup _{n} n^{\frac{1}{p}} x_{\phi(n)}, & 1 \leq p<\infty, q=\infty
\end{array}\right.
$$

is finite, where $\left(x_{\phi(n)}\right)$ is non-increasing rearrangement of $x$. The spaces $\ell_{p, q}$ have been used to introduce and investigate some classes of operators, like $(p, q)$-nuclear, $(p, q ; r)$-absolutely summing operator 14. Kato [11] characterized the dual space of $\ell_{p, q}\{E\}$, where $E$ is a Banach space. See also [2], 10, [15].

The idea of grand spaces was raised by Iwaniec and Sbordone 8 . They introduced the grand Lebesgue spaces $L^{p)}$ for $1<p<\infty$. Samko and Umarkhadzhiev [17] studied some properties of grand Lebesgue spaces on sets of infinite measure. Jain and Kumari [9] introduced the grand Lorentz spaces $\Lambda_{q), w}, 0<q<\infty$ and studied on its basic properties. Also, they characterized boundedness of maximal operator on the space $\Lambda_{q), w}$. Later, Rafeiro and others [16] introduced the grand Lebesgue sequence space $\ell^{p), \theta}=\ell^{p), \theta}(X)$ by the norm

$$
\|x\|_{\ell^{p), \theta}(X)}=\sup _{\varepsilon>0}\left(\varepsilon^{\theta} \sum_{k \in X}\left|x_{k}\right|^{p(1+\varepsilon)}\right)^{\frac{1}{p(1+\varepsilon)}}=\sup _{\varepsilon>0} \varepsilon^{\frac{\theta}{(1+\varepsilon)}}\|x\|_{\ell^{p(1+\varepsilon)}(X)}
$$

where $X$ is one of the sets $\mathbb{Z}^{n}, \mathbb{Z}, \mathbb{N}$ and $\mathbb{N}_{0}$ for $1 \leq p<\infty, \theta>0$. They studied various operators of harmonic analysis, e. g. maximal, convolution, Hardy etc.

In this paper, we are inspired by this work and introduce the grand Lorentz sequence spaces $\ell_{p, q)}^{\theta}$ as follows; let $\theta>0$. The grand Lorentz sequence space $\ell_{p, q)}^{\theta}$ is the set of all sequences $a=\left(a_{n}\right)$ such that $\|a\|_{p, q), \theta}<\infty$, where $\|a\|_{p, q), \theta}$ is defined by

$$
\left\{\begin{array}{cc}
\sup _{\varepsilon>0}\left(\varepsilon^{\theta} \sum_{n=1}^{\infty}\left(n^{\frac{1}{p(1+\varepsilon)}} a_{\phi(n)}\right)^{q(1+\varepsilon)} n^{-1}\right)^{\frac{1}{q(1+\varepsilon)}}, & 1 \leq p \leq \infty, 1 \leq q<\infty \\
\sup _{n \geq 1} n^{\frac{1}{p}} a_{\phi(n)}, & 1 \leq p<\infty, q=\infty
\end{array}\right.
$$

where $\left(a_{\phi(n)}\right)$ is the non-increasing rearrangement of the sequence $a=\left(a_{n}\right)$. In case $p=q$, the grand Lorentz sequence space $\ell_{p, q)}^{\theta}$ coincides with the grand Lebesgue 
space $\ell^{p), \theta}(\mathbb{N})$. In this work, we study on some topological properties and inclusion theorems of the space $\ell_{p, q)}^{\theta}$. Also, we characterize some properties of multiplication operator on the $\ell_{p, q)}^{\theta}$.

We will need the following lemma:

Lemma 1. (Hardy, Littlewood and Polya) Let $\left(r_{n}^{*}\right)$ and $\left({ }^{*} r_{n}\right)$ be the non-increasing and non-decreasing rearrangements of a finite sequence $\left(r_{n}\right)$ of positive numbers. Then, we have for any two sequences $\left(a_{n}\right)$ and $\left(b_{n}\right)$ of positive numbers such that

$$
\sum_{n} a_{n}^{* *} b_{n} \leq \sum_{n} a_{n} b_{n} \leq \sum_{n} a_{n}^{*} b_{n}^{*}
$$

6].

\section{Main Results}

\subsection{Grand Lorentz Sequence Space.}

Theorem 2. The grand Lorentz sequence space $\ell_{p, q)}^{\theta}$ is a normed space for $1 \leq q \leq$ $p \leq \infty$ and a quasi-normed space for $1 \leq p<q \leq \infty$.

Proof. By definition of the norm of $\ell_{p, q)}^{\theta}$, we can write

$$
\|a\|_{p, q), \theta}=\sup _{\varepsilon>0} \varepsilon^{\frac{\theta}{q(1+\varepsilon)}}\|a\|_{p, q(1+\varepsilon)} .
$$

Let $1 \leq q<p \leq \infty$. For any $a, b \in \ell_{p, q)}^{\theta}$, since $n^{\frac{q}{p}-1}$ is decreasing sequence of positive numbers and so by Lemma 1 , we have

$$
\begin{aligned}
& \|a+b\|_{p, q), \theta}=\sup _{\varepsilon>0}\left(\varepsilon^{\theta} \sum_{n=1}^{\infty} n^{\frac{q}{p}-1}\left(a_{\vartheta(n)}+b_{\vartheta(n)}\right)^{q(1+\varepsilon)}\right)^{\frac{1}{q(1+\varepsilon)}} \\
& =\sup _{\varepsilon>0}\left(\varepsilon^{\theta} \sum_{n=1}^{\infty}\left(n^{\left(\frac{q}{p}-1\right) \frac{1}{q(1+\varepsilon)}}\left(a_{\vartheta(n)}+b_{\vartheta(n)}\right)\right)^{q(1+\varepsilon)}\right)^{\frac{1}{q(1+\varepsilon)}} \\
& \leq \sup _{\varepsilon>0}\left(\varepsilon^{\theta} \sum_{n=1}^{\infty} n^{\frac{q}{p}-1}\left(a_{\vartheta(n)}\right)^{q(1+\varepsilon)}\right)^{\frac{1}{q(1+\varepsilon)}} \\
& +\sup _{\varepsilon>0}\left(\varepsilon^{\theta} \sum_{n=1}^{\infty} n^{\frac{q}{p}-1}\left(b_{\vartheta(n)}\right)^{q(1+\varepsilon)}\right)^{\frac{1}{q(1+\varepsilon)}} \\
& \leq \sup _{\varepsilon>0}\left(\varepsilon^{\theta} \sum_{n=1}^{\infty} n^{\frac{q}{p}-1}\left(a_{\phi(n)}\right)^{q(1+\varepsilon)}\right)^{\frac{1}{q(1+\varepsilon)}} \\
& +\sup _{\varepsilon>0}\left(\varepsilon^{\theta} \sum_{n=1}^{\infty} n^{\frac{q}{p}-1}\left(b_{\psi(n)}\right)^{q(1+\varepsilon)}\right)^{\frac{1}{q(1+\varepsilon)}}
\end{aligned}
$$




$$
=\|a\|_{p, q), \theta}+\|b\|_{p, q), \theta}
$$

where $\left(a_{\vartheta(n)}+b_{\vartheta(n)}\right),\left(a_{\phi(n)}\right)$ and $\left(b_{\psi(n)}\right)$ are the non-increasing rearrangements of $\left(a_{n}+b_{n}\right),\left(a_{n}\right)$ and $\left(b_{n}\right)$, respectively.

Let $1 \leq p<q<\infty$. Then, we have $p<q(1+\varepsilon)$ for $\varepsilon>0$ and hence $\|a\|_{p, q(1+\varepsilon)}$ is a quasi-norm. Thus, we get

$$
\begin{aligned}
\|a+b\|_{p, q), \theta} & =\sup _{\varepsilon>0} \varepsilon^{\frac{\theta}{q(1+\varepsilon)}}\|a+b\|_{p, q(1+\varepsilon)} \\
& \leq \sup _{\varepsilon>0} \varepsilon^{\frac{\theta}{q(1+\varepsilon)}}\left(2^{\frac{1}{p}}\left(\|a\|_{p, q(1+\varepsilon)}+\|b\|_{p, q(1+\varepsilon)}\right)\right) \\
& \leq 2^{\frac{1}{p}}\left(\|a\|_{p, q), \theta}+\|b\|_{p, q), \theta}\right) .
\end{aligned}
$$

For $1 \leq p<\infty$ and $q=\infty$, we have $\|a\|_{p, \infty), \theta}=\|a\|_{p, \infty}$. The proof is completed.

Remark 3. Let $\alpha>0$ and let us take the sequence

$$
\left(a_{n}\right)=\left(n^{\frac{-1}{p}}(\ln (n+1))^{-\alpha}\right)
$$

as in [16]. It is easy to see that the sequence $\left(a_{n}\right)$ is decreasing and thus the nonincreasing rearrangement of $\left(a_{n}\right)$ is itself. Therefore, we have

$$
\sum_{n=1}^{\infty}\left(n^{\frac{1}{p}} n^{\frac{-1}{p}}(\ln (n+1))^{-\alpha}\right)^{q} n^{-1}=\sum_{n=1}^{\infty} n^{-1}(\ln (n+1))^{-\alpha q} .
$$

If $\alpha>\frac{1}{q}$, then $\left(a_{n}\right) \in \ell_{p, q}$. Using similar technique as in [16], we get $\left(a_{n}\right) \in \ell_{p, q)}^{\theta}$ if and only if $\alpha \geq \frac{1-\theta}{q}$. Thus, we get $\left(a_{n}\right) \in \ell_{p, q)}^{\theta}$ and $\left(a_{n}\right) \notin \ell_{p, q}$ whenever $\frac{1-\theta}{q} \leq \alpha \leq \frac{1}{q}$.

Definition 4. The vanishing grand Lorentz sequence space $\dot{\ell}_{p, q)}^{\theta}, 1 \leq p \leq \infty, 1 \leq$ $q<\infty$, consists of all sequences $\left(a_{n}\right) \in \ell_{p, q)}^{\theta}$ such that

$$
\lim _{\varepsilon \rightarrow 0} \varepsilon^{\theta} \sum_{n=1}^{\infty}\left(n^{\frac{1}{p(1+\varepsilon)}} a_{\phi(n)}\right)^{q(1+\varepsilon)} n^{-1}=0 .
$$

Lemma 5. The space $\ell_{p, q)}^{\theta}$ is a closed subspace of the space $\ell_{p, q)}^{\theta}$.

Proof. The proof can be obtained by using similar technique as in [16].

Remark 6. It is enough to take the supremum in (1) on the finite interval for $\varepsilon$, which means

$$
\|a\|_{p, q), \theta}=\sup _{0<\varepsilon<\frac{1}{W(1 / e)}} \varepsilon^{\frac{\theta}{q(1+\varepsilon)}}\|a\|_{p, q(1+\varepsilon)}
$$

where $W(t)$ is the Lambert function. Note that $\frac{1}{W(1 / e)} \approx 3.59$ (see [4, [16]). 
Lemma 7. Let $a=\left(a_{n}\right) \in \ell_{p, q)}^{\theta}, 1 \leq p, q<\infty$ and $\theta>0$. Then, we have the following inequalities for all $n \in \mathbb{N}$ :

$$
a_{\phi(n)} \leq h\left(\frac{1}{W\left(e^{-1}\right)}\right)^{\frac{-\theta}{q}}\left(\frac{p}{q} R\left(\varepsilon_{0}\right)\right)^{\frac{-1}{q}} n^{\frac{-1}{p}}\|a\|_{p, q), \theta}
$$

if $1 \leq p \leq q<\infty$ and

$$
a_{\phi(n)} \leq h\left(\frac{1}{W\left(e^{-1}\right)}\right)^{-\frac{\theta}{q}} n^{\frac{1}{q}-\frac{1}{p}}\|a\|_{p, q), \theta}
$$

if $1 \leq q<p \leq \infty$, where $h(x)=x^{\frac{1}{1+x}}, R(x)=(1+x)^{-\frac{1}{1+x}}$ and $\varepsilon_{0} \approx 1,7182$.

Proof. Let $a=\left(a_{n}\right) \in \ell_{p, q)}^{\theta}$ and let $1 \leq p \leq q<\infty$. Since $p \leq q(1+\varepsilon)$, we have by Lemma 2 in [1] that

$$
\begin{aligned}
\|a\|_{p, q), \theta} & =\sup _{0<\varepsilon<\frac{1}{W\left(e^{-1}\right)}} h(\varepsilon)^{\frac{\theta}{q}}\|a\|_{p, q(1+\varepsilon)} \\
& \geq \sup _{0<\varepsilon<\frac{1}{W\left(e^{-1}\right)}} h(\varepsilon)^{\frac{\theta}{q}}\left(n^{\frac{1}{p}}\left(\frac{p}{q(1+\varepsilon)}\right)^{\frac{1}{q(1+\varepsilon)}} a_{\phi(n)}\right) \\
& \geq \sup _{0<\varepsilon<\frac{1}{W\left(e^{-1}\right)}} h(\varepsilon)^{\frac{\theta}{q}}\left(\frac{p}{q}\right)^{\frac{1}{q}}(1+\varepsilon)^{-\frac{1}{q(1+\varepsilon)}} n^{\frac{1}{p}} a_{\phi(n)} \\
& =\sup _{0<\varepsilon<\frac{1}{W\left(e^{-1}\right)}} h(\varepsilon)^{\frac{\theta}{q}}\left(\frac{p}{q}\right)^{\frac{1}{q}}(R(\varepsilon))^{\frac{1}{q}} n^{\frac{1}{p}} a_{\phi(n)} . \\
& \geq \sup _{0<\varepsilon<\frac{1}{W\left(e^{-1}\right)}} h(\varepsilon)^{\frac{\theta}{q}}\left(\frac{p}{q}\right)^{\frac{1}{q}}\left(R\left(\varepsilon_{0}\right)\right)^{\frac{1}{q}} n^{\frac{1}{p}} a_{\phi(n)} . \\
& =h\left(\frac{1}{W\left(e^{-1}\right)}\right)^{\frac{\theta}{q}}\left(\frac{p}{q}\right)^{\frac{1}{q}}\left(R\left(\varepsilon_{0}\right)\right)^{\frac{1}{q}} n^{\frac{1}{p}} a_{\phi(n)} .
\end{aligned}
$$

Here $R(x)=(1+x)^{-\frac{1}{1+x}}$ attains the minimum at the point $\varepsilon_{0} \approx 1,7182$.

Let $1 \leq q<p<\infty$. Then, since $n^{\frac{q}{p}-1}$ is decreasing, we have

$$
\begin{aligned}
\|a\|_{p, q), \theta} & =\sup _{0<\varepsilon<\frac{1}{W\left(e^{-1}\right)}} h(\varepsilon)^{\frac{\theta}{q}}\|a\|_{p, q(1+\varepsilon)} \\
& \geq \sup _{0<\varepsilon<\frac{1}{W\left(e^{-1}\right)}} h(\varepsilon)^{\frac{\theta}{q}}\left(\sum_{n=1}^{k}\left(n^{\frac{1}{p(1+\varepsilon)}} a_{\phi(n)}\right)^{q(1+\varepsilon)} n^{-1}\right)^{\frac{1}{q(1+\varepsilon)}} \\
& \geq a_{\phi(k)} \sup _{0<\varepsilon<\frac{1}{W\left(e^{-1}\right)}} h(\varepsilon)^{\frac{\theta}{q}}\left(\sum_{n=1}^{k} n^{\frac{q}{p}-1}\right)^{\frac{1}{q(1+\varepsilon)}}
\end{aligned}
$$




$$
\begin{aligned}
& \geq a_{\phi(k)} \sup _{0<\varepsilon<\frac{1}{W\left(e^{-1}\right)}} h(\varepsilon)^{\frac{\theta}{q}}\left(k^{\frac{q}{p}-1}\right)^{\frac{1}{q(1+\varepsilon)}} \\
& \geq h\left(\frac{1}{W\left(e^{-1}\right)}\right)^{\frac{\theta}{q}} n^{\frac{1}{p}-\frac{1}{q}} a_{\phi(k)} .
\end{aligned}
$$

Theorem 8. The space $\ell_{p, q)}^{\theta}$ is complete for $1 \leq p, q \leq \infty$.

Proof. Let $a^{(s)}=\left(a_{n}^{(s)}\right) \in \ell_{p, q)}^{\theta}$ such that

$$
\lim _{s, t \rightarrow \infty}\left\|a^{(s)}-a^{(t)}\right\|_{p, q), \theta}=0 .
$$

For $q=\infty$, the proof is clear. Let $q<\infty$. Then, there exists a natural number $s_{0}$ such that

$$
\left\|a^{(s)}-a^{(t)}\right\|_{p, q), \theta}<\eta
$$

whenever $s, t \geq s_{0}$. By Lemma 3 , we have

$$
\begin{aligned}
\left|a_{k}^{(s)}-a_{k}^{(t)}\right| & \leq h\left(\frac{1}{W\left(e^{-1}\right)}\right)^{-\frac{\theta}{q}} \begin{cases}k^{\frac{1}{q}-\frac{1}{p}}\left\|a^{(s)}-a^{(t)}\right\|_{p, q), \theta}, & q<p \\
\left(\frac{p}{q} R\left(\varepsilon_{0}\right)\right)^{-\frac{1}{q}} k^{-\frac{1}{p}}\left\|a^{(s)}-a^{(t)}\right\|_{p, q), \theta}, & p \leq q\end{cases} \\
& <h\left(\frac{1}{W\left(e^{-1}\right)}\right)^{-\frac{\theta}{q}} \begin{cases}k^{\frac{1}{q}-\frac{1}{p}} \eta, & q<p \\
\left(\frac{p}{q} R\left(\varepsilon_{0}\right)\right)^{-\frac{1}{q}} k^{-\frac{1}{p}} \eta, & p \leq q\end{cases}
\end{aligned}
$$

where $h(x)=x^{\frac{1}{1+x}}, R(x)=(1+x)^{-\frac{1}{1+x}}$. This shows that $\left(a_{k}^{(s)}\right)$ is a Cauchy sequence in $\mathbb{C}$. Thus, we have $\left(a_{k}\right) \in \mathbb{C}$ such that $\lim _{s \rightarrow \infty}\left|a_{k}^{(s)}-a_{k}\right|=0$. By using the equality (1) with classical method, we get $\ell_{p, q)}^{\theta}$ is a complete space.

Lemma 9. Let $1 \leq p<\infty, 1 \leq q<q_{1} \leq \infty$. Then, we have the following

$$
\ell_{p, q)}^{\theta} \subset \ell_{\left.p, q_{1}\right)}^{\theta} .
$$

Proof. Let $a=\left(a_{n}\right) \in \ell_{p, q)}^{\theta}$ and $p<q$. Then, we have by Proposition 2 in [1] that

$$
\begin{aligned}
\|a\|_{\left.p, q_{1}\right), \theta} & =\sup _{0<\varepsilon<\frac{1}{W\left(e^{-1}\right)}} h(\varepsilon)^{\frac{\theta}{q_{1}}}\|a\|_{p, q_{1}(1+\varepsilon)} \\
& \leq \sup _{0<\varepsilon<\frac{1}{W\left(e^{-1}\right)}} h(\varepsilon)^{\frac{\theta}{q_{1}}}\left(\frac{q(1+\varepsilon)}{p}\right)^{\frac{1}{q(1+\varepsilon)}-\frac{1}{q_{1}}}\|a\|_{p, q(1+\varepsilon)} \\
& \leq\left(\frac{q}{p}\left(1+\frac{1}{W\left(e^{-1}\right)}\right)\right)^{\frac{1}{q}-\frac{1}{q_{1}}}\|a\|_{p, q), \theta} \\
& <\infty
\end{aligned}
$$


where $h(x)=x^{\frac{1}{1+x}}$. The inclusion can be obtained by similar way for $p \geq q$ with Lemma 3.

Theorem 10. Let either $1 \leq p<p_{1} \leq \infty, 1 \leq q<\infty$ or $1 \leq p<p_{1}<\infty, q=\infty$. Then, the inclusion

$$
\ell_{p, q)}^{\theta} \subset \ell_{\left.p_{1}, q\right)}^{\theta}
$$

holds.

Proof. Let $a \in \ell_{p, q)}^{\theta}$. Then, we have

$$
\begin{aligned}
\|a\|_{\left.p_{1}, q\right), \theta} & =\sup _{0<\varepsilon<\frac{1}{W\left(e^{-1}\right)}} h(\varepsilon)^{\frac{\theta}{q}}\|a\|_{p_{1}, q(1+\varepsilon)} \\
& \leq \sup _{0<\varepsilon<\frac{1}{W\left(e^{-1}\right)}} h(\varepsilon)^{\frac{\theta}{q}}\|a\|_{p, q(1+\varepsilon)} \\
& =\|a\|_{p, q), \theta} \\
& <\infty
\end{aligned}
$$

which shows $a \in \ell_{\left.p_{1}, q\right)}^{\theta}$.

Corollary 11. Let $1 \leq p_{1}<p \leq q<q_{1} \leq \infty$. Then, the inclusions

$$
\ell^{\left.p_{1}\right), \theta} \subset \ell_{p, q)}^{\theta} \subset \ell^{\left.q_{1}\right), \theta}
$$

hold.

Theorem 12. The grand Lorentz sequence space $\ell_{p, q)}^{\theta}$ is strictly convex for $1<$ $p<\infty$ and $1<q<\infty$.

Proof. Let $a, b \in \ell_{p, q)}^{\theta}$ such that $\|a\|_{p, q), \theta}=\|b\|_{p, q), \theta}=1$ and $\left\|\frac{a+b}{2}\right\|_{p, q), \theta}=1$. Then, we have by using similar technique as in [1] that

$$
\begin{aligned}
1 & =\left\|\frac{a+b}{2}\right\|_{p, q), \theta}=\sup _{0<\varepsilon<\frac{1}{W\left(e^{-1}\right)}} \varepsilon^{\frac{\theta}{q(1+\varepsilon)}}\left\|\frac{a+b}{2}\right\|_{p, q(1+\varepsilon)} \\
& \leq \sup _{0<\varepsilon<\frac{1}{W\left(e^{-1}\right)}} \varepsilon^{\frac{\theta}{q(1+\varepsilon)}}\left(\frac{\|a\|_{p, q(1+\varepsilon)}+\|b\|_{p, q(1+\varepsilon)}}{2}\right) \\
& \leq\left(\frac{\|a\|_{p, q), \theta}+\|b\|_{p, q), \theta}}{2}\right) \\
& =1
\end{aligned}
$$

which shows $a=b$. 
2.2. Multiplication Operator. In this section, we characterize some properties of the multiplication operators on $\ell_{p, q)}^{\theta}$. Let $v=\left(v_{n}\right)$ be a complex-valued sequence and let us define the linear transformation $M_{v}$ on the sequence space $X$ into the linear space of all complex-valued sequences by

$$
M_{v}(x)=v x=\left(v_{n} x_{n}\right) .
$$

If the linear transformation $M_{v}$ is bounded with range in $X$, then it is called multiplication operator on $X$.

Theorem 13. Let $v=\left(v_{n}\right)$ be a complex-valued sequence. Then, $M_{v}$ is a multiplication operator on $\ell_{p, q)}^{\theta}, 1 \leq p, q \leq \infty$ if and only if $v$ is a bounded sequence.

Proof. Let $M_{v}$ be a multiplication operator on $\ell_{p, q)}^{\theta}$ and let $q<\infty$. Then, there exists a positive number $K>0$ such that

$$
\left\|M_{v}(a)\right\|_{p, q), \theta} \leq K\|a\|_{p, q), \theta}
$$

for all $a \in \ell_{p, q)}^{\theta}$. Let us define

$$
e_{n}^{(k)}=\left\{\begin{array}{cc}
s^{-\frac{\theta}{p}}, & k=n \\
0, & k \neq n
\end{array}\right.
$$

where $s=\left(\frac{1}{W\left(e^{-1}\right)}\right)^{\frac{W\left(e^{-1}\right)}{1+W\left(e^{-1}\right)}}$ for all $n \in \mathbb{N}$. Then, the non-increasing rearrangement of $\left(e_{n}^{(k)}\right)$ is

$$
e_{\phi(n)}^{(k)}=\left\{\begin{array}{cc}
s^{-\frac{\theta}{p}} & , n=1 \\
0 & , n \neq 1
\end{array} .\right.
$$

Then, we have $\left(e_{n}^{(k)}\right) \in \ell_{p, q)}^{\theta}$ with $\left\|e^{(k)}\right\|_{p, q), \theta}=1$. By the boundedness of $M_{v}$, it can be written $\left\|M_{v} e^{(k)}\right\|_{p, q), \theta} \leq K\left\|e^{(k)}\right\|_{p, q), \theta}=K$. Thus, we get

$$
\begin{aligned}
\sup _{\varepsilon>0}\left(\varepsilon^{\theta} \sum_{n=1}^{\infty}\left(n^{\frac{1}{p(1+\varepsilon)}} v_{\psi(n)} e_{\psi(n)}^{(k)}\right)^{q(1+\varepsilon)} n^{-1}\right)^{\frac{1}{q(1+\varepsilon)}} & =\sup _{\varepsilon>0}\left(\varepsilon^{\theta}\left(v_{\psi(1)} e_{\psi(1)}^{(k)}\right)^{q(1+\varepsilon)}\right)^{\frac{1}{q(1+\varepsilon)}} \\
& =s^{-\frac{\theta}{p}} \sup _{\varepsilon>0}\left(\varepsilon^{\frac{\theta}{q(1+\varepsilon)}} v_{\psi(1)}\right) \\
& \leq K
\end{aligned}
$$

which gives that $v_{\psi(1)} \leq K . s^{-\frac{\theta}{q}+\frac{\theta}{p}}$. This shows that $v$ is bounded. If $q=\infty$, the proof is similar as was used in the classical Lorentz sequence spaces.

Conversely, let $v$ be a bounded sequence. Then, there exists $T>0$ such that $\left|v_{k}\right| \leq T$ for all $k \in \mathbb{N}$. Thus, we get

$$
\left\|M_{v} a\right\|_{p, q), \theta}=\sup _{\varepsilon>0}\left(\varepsilon^{\theta} \sum_{k=1}^{\infty}\left(k^{\frac{1}{p(1+\varepsilon)}} v_{\psi(k)} a_{\psi(k)}\right)^{q(1+\varepsilon)} k^{-1}\right)^{\frac{1}{q(1+\varepsilon)}}
$$




$$
\begin{aligned}
& \leq T \sup _{\varepsilon>0}\left(\varepsilon^{\theta} \sum_{k=1}^{\infty}\left(k^{\frac{1}{p(1+\varepsilon)}} a_{\psi(k)}\right)^{q(1+\varepsilon)} k^{-1}\right)^{\frac{1}{q(1+\varepsilon)}} \\
& =T\|a\|_{p, q), \theta}
\end{aligned}
$$

for $q<\infty$. If $q=\infty$, then

$$
\sup _{k \in \mathbb{N}} k^{\frac{1}{p}} v_{\psi(k)} a_{\psi(k)} \leq T\|a\|_{p, q), \theta} .
$$

Theorem 14. Let $M_{v}$ be a multiplication operator on $\ell_{p, q)}^{\theta}, 1 \leq p, q \leq \infty$. Then, $M_{v}$ is invertible if and only if there exists $\mu>0$ such that $\left|v_{n}\right| \geq \mu . s^{-\frac{\theta}{q}+\frac{2 \theta}{p}}$, where $s=\left(\frac{1}{W\left(e^{-1}\right)}\right)^{\frac{W\left(e^{-1}\right)}{1+W\left(e^{-1}\right)}}$ for all $n \in \mathbb{N}$.

Proof. Let $M_{v}$ be invertible operator on $\ell_{p, q)}^{\theta}, 1 \leq p, q \leq \infty$. Then, there exists $\rho>0$ such that

$$
\left\|M_{v} a\right\|_{p, q), \theta} \geq \mu\|a\|_{p, q), \theta}
$$

for all $a \in \ell_{p, q)}^{\theta}$. Thus, for $\left(e_{n}^{(k)}\right) \in \ell_{p, q)}^{\theta}$, we get

$$
\left\|M_{v} e^{(k)}\right\|_{p, q), \theta}=s^{\frac{\theta}{q}-\frac{\theta}{p}}\left|v_{k}\right| \geq \mu s^{\frac{\theta}{p}}
$$

which gives $\left|v_{k}\right| \geq s^{-\frac{\theta}{q}+\frac{2 \theta}{p}} \mu$. Conversely, let define $z_{k}=\left(v_{k}\right)^{-1}$. By using Theorem 5 , the proof can be obtained.

Theorem 15. Let $M_{v}$ be a multiplication operator on $\ell_{p, q)}^{\theta}, 1 \leq p, q \leq \infty$. Then, a necessary and sufficient condition for $M_{v}$ to have closed range is that for some $\varrho>0$

$$
\left|v_{n}\right| \geq \varrho
$$

for each $n \in R=\left\{n \in \mathbb{N}: v_{n} \neq 0\right\}$.

Proof. Assume that $\left|v_{n}\right| \geq \varrho$ for $\varrho>0$ and for all $n \in R$. Let $q<\infty$ and let $g^{(k)}, g \in \ell_{p, q)}^{\theta}$ such that $M_{v} g^{(k)} \rightarrow g$ as $k \rightarrow \infty$. Then, we write

$$
\lim _{m, n \rightarrow \infty}\left\|M_{v} g^{(m)}-M_{v} g^{(n)}\right\|_{p, q), \theta}=0 .
$$

Put $x^{(m n)}=g^{(m)}-g^{(n)}$. Thus, we have

$$
\left\{l \in \mathbb{N}:\left|x_{l}^{(m n)}\right|>\frac{r}{\varrho}\right\} \subseteq\left\{l \in \mathbb{N}:\left|v_{l} x_{l}^{(m n)}\right|>r\right\}
$$

for each $r>0$ and so $\varrho x_{\phi(l)}^{(m n)} \leq v_{\psi(l)} x_{\psi(l)}^{(m n)}$, where $x_{\phi(l)}^{(m n)}$ and $v_{\psi(l)} x_{\psi(l)}^{(m n)}$ are the nonincreasing rearrangement of the sequences $\left(x_{l}^{(m n)}\right)$ and $\left(v_{l} x_{l}^{(m n)}\right)$, respectively. 
Thus, we have

$$
\begin{aligned}
\left\|v x^{(m n)}\right\|_{p, q), \theta} & =\left\|M_{v} g^{(m)}-M_{v} g^{(n)}\right\|_{p, q), \theta} \\
& =\sup _{\varepsilon>0}\left(\varepsilon^{\theta} \sum_{l \in R}\left(l^{\frac{1}{p(1+\varepsilon)}} v_{\psi(l)} x_{\psi(l)}^{(m n)}\right)^{q(1+\varepsilon)} l^{-1}\right)^{\frac{1}{q(1+\varepsilon)}} \\
& \geq \sup _{\varepsilon>0}\left(\varepsilon^{\theta} \sum_{l \in R} \varrho^{q(1+\varepsilon)}\left(l^{\frac{1}{p(1+\varepsilon)}} x_{\phi(l)}^{(m n)}\right)^{q(1+\varepsilon)} l^{-1}\right)^{\frac{1}{q(1+\varepsilon)}} \\
& =\varrho\left\|x^{(m n)}\right\|_{p, q), \theta} .
\end{aligned}
$$

Since $\left\|v x^{(m n)}\right\|_{p, q), \theta} \rightarrow 0$ as $m, n \rightarrow \infty$, we have $x^{(m n)} \rightarrow 0$ as $m, n \rightarrow \infty$. This means that $g^{(m)}$ is a Cauchy sequence in $\left.\ell_{p, q)}^{\theta}\right|_{R}$, where $\left.\ell_{p, q)}^{\theta}\right|_{R}=\left\{a=\left(a_{k}\right) \in \ell_{p, q)}^{\theta}: a_{k}=0\right.$ if $\left.k \in \mathbb{N} \backslash R\right\}$ is a closed subspace of $\ell_{p, q)}^{\theta}$. Thus, we get $\left.f \in \ell_{p, q)}^{\theta}\right|_{R}$ such that $g^{(m)} \rightarrow f$ as $m \rightarrow \infty$. Since $M_{v}$ is bounded linear operator, we can write $M_{v} g^{(m)} \rightarrow M_{v} f$. This gives $M_{v} f=g$. Because of $\operatorname{Ker}\left(M_{v}\right)=\left.\ell_{p, q}^{\theta}\right|_{\mathbb{N} \backslash R}, M_{v}$ has closed range.

Conversely, assume that $M_{v}$ has closed range and there exists $\left(l_{n}\right) \in R$ such that $\left|v_{l_{n}}\right|<\frac{1}{n}$. Let

$$
e_{m}^{\left(l_{n}\right)}=\left\{\begin{array}{cc}
s^{-\frac{\theta}{p}}, & m=l_{n} \\
0, & m \neq l_{n}
\end{array}\right.
$$

where $s=\left(\frac{1}{W\left(e^{-1}\right)}\right)^{\frac{W\left(e^{-1}\right)}{1+W\left(e^{-1}\right)}}$ and let $q<\infty$. Then, $\left\|e^{\left(l_{n}\right)}\right\|_{p, q), \theta}=1$. Thus, we get

$$
\begin{aligned}
\left\|M_{v} e^{\left(l_{n}\right)}\right\|_{p, q), \theta} & =\left\|v e^{\left(l_{n}\right)}\right\|_{p, q), \theta} \\
& =\sup _{\varepsilon>0}\left(\varepsilon^{\theta} \sum_{m=1}^{\infty}\left(m^{\frac{1}{p(1+\varepsilon)}} v_{\psi(m)} e_{\psi(m)}^{\left(l_{n}\right)}\right)^{q(1+\varepsilon)} m^{-1}\right)^{\frac{1}{q(1+\varepsilon)}} \\
& =\sup _{\varepsilon>0}\left(\varepsilon^{\theta}\left(v_{\psi(1)} e_{\psi(1)}^{\left(l_{n}\right)}\right)^{q(1+\varepsilon)}\right)^{\frac{1}{q(1+\varepsilon)}} \\
& =s^{\frac{\theta}{p}-\frac{\theta}{q}} v_{l_{n}} \\
& <\frac{1}{n} s^{\frac{\theta}{p}-\frac{\theta}{q}}\left\|e^{\left(l_{n}\right)}\right\|_{p, q), \theta}
\end{aligned}
$$

which means $M_{v}$ is not bounded different from zero. Thus, $\left|v_{n}\right| \geq \varrho$ for some $\varrho>0$ and all $n \in R$. For the case $q=\infty$ the proof can be obtained by similar way.

Theorem 16. Let $M_{v}$ be a multiplication operator on $\ell_{p, q)}^{\theta}$. Then, $M_{v}$ is compact if and only if $\left|v_{n}\right| \rightarrow 0$ as $n \rightarrow \infty$. 
Proof. The proof can be obtained by the similar way used in the classical Lorentz sequence space.

Corollary 17. Let $M_{v}$ be a multiplication operator on $\ell_{p, q)}^{\theta}$. Then, $M_{v}$ is Fredholm if and only if the set $\mathbb{N} \backslash R$ has finite elements and there exists $\rho>0$ such that

$$
\left|v_{n}\right| \geq \varrho
$$

for all $n \in \mathbb{N}$, where $R=\left\{n \in \mathbb{N}: v_{n} \neq 0\right\}$.

\section{REFERENCES}

[1] Altshuler, Z., Uniform convexity in Lorentz sequence spaces, Isr. J. Math., 20, (1975), 3-4.

[2] Arora, S.C., Datt, G. and Verma, S., Operators on Lorentz sequence spaces, Math. Bohem., No:1, (2009), 87-98.

[3] Bennett, C. and Sharpley, R., Interpolation Operators, Academic Press Inc. Toronto, 1988.

[4] Corless, R. M., Gonnet, G. H., Hare, D. E. G., Jeffrey, D. J. and Knuth, E., On the Lambert W function, Adv. Comput. Math., 5(4), (1996), 329-359.

[5] Crowe, J. A., Zweibel, J. A and Rosenbloom, P. C., Rearrangement of functions, J. Funct. Anal., 66, (1986), 432-438.

[6] Hardy, G. H., Littlewood, J. E. and Polya, G., Inequalities, Cambridge Univ. Press, 1967.

[7] Hunt, R. A., On L(p,q) spaces, Enseign. Math., 12, (1966), 249-276.

[8] Iwaniec, T. and Sbordone, C., On the integrability of the Jacobian under minimal hypotheses, Arch. Ration. Mech. Anal., 119(2), (1992), 129-143.

[9] Jain, P. and Kumari, S., On grand Lorentz spaces and the maximal operator, Math. Student, $79,2010$.

[10] Kaminska, A. and Raynaud, Y., Isomorphic $l_{p}$-subspaces in Orlicz-Lorentz sequence spaces, Proc. Amer. Math. Soc., 134, (2006), 2317-2327.

[11] Kato, M., On Lorentz spaces, Hiroshima Math. J., 6, (1976), 73-93.

[12] Lorentz, G. G., Some new functional spaces, Ann. of Math, 2(51), (1950), 37-55.

[13] Lorentz, G. G., On the theory of spaces $\Lambda$, Pacific J. Math., 1, (1951), 411-429.

[14] Miyazaki, K., $(p, q)$-nuclear and $(p, q)$-integral operators, Hiroshima Math. J., 4, (1974), 99132.

[15] Oğur, O. and Duyar, C., On generalized Lorentz sequence space defined by modulus functions, Filomat, 30(2), (2016), 497-504.

[16] Rafeiro, H., Samko, S. and Umarkhadzhiev, S., Grand Lebesgue sequence spaces, Georgian Math. J., 19(2), (2018), 235-246.

[17] Samko, S. and Umarkhadzhiev, S., On grand Lebesgue spaces on sets of infinite measure, Math. Nachr., 290, (2017), 913-919.

Current address, O.Oğur: Giresun University, Faculty of Education, Department of Elementary Mathematics, Giresun, Turkey

E-mail address: oguz.ogur@giresun.edu.tr

ORCID Address: http://orcid.org/0000-0002-3206-5330 